\title{
Facteurs associés à la lombalgie chez les hommes salariés des Pays de la Loire
}

\author{
Factors associated with low back pain among male employees in Pays de la Loire
}

\author{
Ramond $\mathrm{A}^{1}$, Bodin $\mathrm{J}^{2}$, Sérazin $\mathrm{C}^{2}$, Parot $\mathrm{E}^{3}$, Petit Le Manach $\mathrm{A}^{4}$, Roquelaure $\mathrm{Y}^{5}$
}

\begin{abstract}
Résumé
Objectif : Les lombalgies ont des répercussions majeures sur le monde du travail. Un manque de données prospectives et représentatives de la diversité des emplois limite l'analyse des facteurs de risque chez les travailleurs. L'objectif de cette étude était d'estimer, à l'aide d'une modélisation, les facteurs associés à la lombalgie chez les salariés des Pays de la Loire.

Méthodes : Un échantillon aléatoire de salariés a fait l'objet de deux enquêtes successives. La prévalence des lombalgies lors de la deuxième enquête a été modélisée, par régression logistique multi-étapes, à partir de facteurs de risque individuels et professionnels recueillis lors de la première enquête. Des analyses de sensibilité ont été réalisées. Les analyses ont été stratifiées par sexe ; seuls les résultats pour les hommes sont présentés.

Résultats : Parmi les 3710 participants à la première enquête, 2332 ont répondu à la deuxième enquête, dont 1313 hommes. La prévalence des lombalgies était de $30 \%$ chez les hommes. Des douleurs du cou ou des membres supérieurs, se pencher fréquemment et travailler plus que prévu étaient les facteurs de risque principaux de lombalgie ultérieure. L'association observée entre un faible soutien de la hiérarchie et des lombalgies ultérieures devenait non significative dans une analyse restreinte aux salariés sans lombalgie lors de la première enquête.
\end{abstract}

Conclusions : Le rôle des facteurs biomécaniques et organisationnels semble prédominant sur celui des facteurs psychosociaux. Celui des facteurs individuels est d'interprétation délicate. Quelques limites méthodologiques doivent être discutées, mais l'analyse simultanée de multiples facteurs dans une population diversifiée de travailleurs constitue un apport original et utile pour des actions de prévention.

Prat Organ Soins. 2011;42(3):157-68

Mots-clés : Lombalgie; exposition professionnelle; prévalence; facteurs de risque ; modèles logistiques.

\section{Summary}

Aim: Low back pain (LBP) has a major impact on work. A lack of prospective data dealing with the diversity of jobs limits the analysis of risk factors for workers. The objective of this study was the prospective modeling of risk of LBP in the salaried workers of the Pays de la Loire region.

Methods: Two successive surveys were conducted in a random sample of salaried workers. The association between individual or occupational risk factors recorded in the first survey and the prevalence of LBP in the second survey was studied, using multi-step logistic regression models. Sensitivity analyses were conducted. The analyses were stratified by sex; only the results related to men are presented.

Results: Among the 3710 participants in the first survey, 2332 responded to the second one, which included 1313 men. The prevalence of LBP was 30\% in men. Neck or upper limbs pain, frequent bending or twisting of the trunk and working more than planned were the main risk factors of later LBP. The association observed between a low supervisor support and LBP were no more significant in an analysis restricted to men without prevalent LBP in the first survey.

Conclusion: The role of the biomechanical and organizational factors was higher than the role played by psychosocial factors. The impact of individual factors is tricky to interpret. Some methodological limitations have to be discussed, but the simultaneous analysis of numerous factors in a varied panel of workers has generated an original and useful contribution for prevention actions.

Prat Organ Soins. 2011;42(3):157-68

Keywords: Low back pain; occupational exposure; prevalence; risk factors; logistic models.

\footnotetext{
1 Médecin généraliste, doctorante en épidémiologie. Laboratoire d'ergonomie et d'épidémiologie en santé au travail (LEEST), EA 4336, Unité associée à 1'Institut de veille sanitaire (InVS), Université d'Angers. Département de médecine générale, Faculté de médecine d'Angers.

2 Statisticienne. Laboratoire d'ergonomie et d'épidémiologie en santé au travail (LEEST), EA 4336, Unité associée à l'InVS, Université d'Angers.

3 Praticien hospitalier. Laboratoire d'ergonomie et d'épidémiologie en santé au travail (LEEST), EA 4336, Unité associée à l'InVS, Université d'Angers. Centre de recherche clinique, CHU d'Angers.

4 Assistante hospitalo-universitaire. Laboratoire d'ergonomie et d'épidémiologie en santé au travail (LEEST), EA 4336, Unité associée à l'InVS, Université d'Angers. Département de santé au travail, CHU d'Angers.

5 Professeur des universités, praticien hospitalier. Laboratoire d'ergonomie et d'épidémiologie en santé au travail (LEEST), EA 4336, Unité associée à 1'InVS, Université d'Angers. Département de santé au travail, CHU d'Angers.

Adresse pour correspondance : $D^{r}$ Aline Ramond. Laboratoire d'ergonomie et d'épidémiologie en santé au travail (LEEST) - UA InVS, Médecine E - CHU d'Angers,

4 rue Larrey, F-49933 Angers Cedex.

E-mail : aline.ramond@univ-angers.fr
} 


\section{INTRODUCTION}

Les lombalgies sont définies comme la perception d'une douleur dans le bas du dos. S'il existe des étiologies spécifiques à certaines lombalgies (processus inflammatoire, tumeur, fracture vertébrale, etc.), on ne retrouve pas de cause précise pour une grande majorité d'entre elles, alors qualifiées de lombalgies communes ou non spécifiques [1].

Les lombalgies communes sont fréquentes. Elles engendrent un coût humain, médical, et socio-économique important. La prévalence annuelle des lombalgies chez les adultes de 30 à 64 ans en population générale était estimée à $54 \%$ chez les hommes et $57 \%$ chez les femmes dans une enquête française de 2002 [2]. Les lombalgies génèrent un fréquent recours aux soins [3], et sont responsables d'un absentéisme important sur le lieu de travail. Le nombre de demandes de reconnaissance en maladie professionnelle ou en accident du travail augmente chaque année en France. Les coûts directs et indirects induits par cette pathologie sont donc majeurs $[4,5]$.

Malgré l'intérêt croissant qui leur est porté, et la multiplication des travaux de recherche ces dernières décennies, la fréquence des lombalgies reste difficile à déterminer. Les estimations de prévalence sont très variables selon les définitions choisies et les populations étudiées $[6,7]$. Par ailleurs, la fréquence très élevée dans la population et l'évolution récurrente des lombalgies rendent difficilement applicable, voire inapproprié, le concept d'incidence [8].

La recherche de facteurs de risque de survenue des lombalgies a fait l'objet de nombreux travaux, notamment dans des populations de travailleurs. Ce milieu est particulièrement étudié car de nombreuses contraintes professionnelles ont été incriminées dans l'apparition ou l'aggravation de ces troubles. De plus, une grande partie des répercussions socio-économiques des lombalgies se concentre sur le monde du travail. Enfin, la mise en évidence de facteurs de risque professionnels modifiables pourrait conduire à des actions de prévention collective en milieu du travail.

La majorité de ces études est transversale, ce qui en limite l'interprétation en termes de causalité. Les études longitudinales sont moins nombreuses, du fait de la difficulté à constituer et à suivre dans le temps des cohortes de travailleurs. Les études expérimentales sur des modifications de contraintes professionnelles sont rares, et les résultats souvent évalués à trop court terme, du fait de contraintes sociales spécifiques imposées par le milieu du travail (mobilité des salariés, changement de direction, restructurations, changements d'activité, etc.).

Une partie des études ne s'intéresse qu'à un nombre restreint de facteurs de risque, voire à un facteur unique, alors que cette pathologie est multifactorielle. D'autres ciblent des catégories spécifiques de travailleurs, et leurs résultats sont difficilement extrapolables à d'autres populations.

La responsabilité de certains facteurs, notamment biomécaniques (port de charges lourdes, mouvements de flexion et torsion du rachis, vibrations dites « corpsentier »), dans la survenue de lombalgies, semble assez largement admise [9, 10]. Néanmoins, l'estimation précise du niveau de risques lié à ces facteurs reste délicate. D'autres facteurs, notamment individuels et psychosociaux, ont un rôle plus controversé dans l'apparition des lombalgies, et restent difficiles à évaluer [11].

La mise en place d'un réseau de médecins du travail dans les Pays de la Loire entre 2002 et 2005 a permis la constitution d'un échantillon aléatoire de travailleurs, conçu pour être représentatif des salariés de la région. Cet échantillon a fait l'objet d'une étude, répétée dans le temps, de la prévalence des principaux troubles musculo-squelettiques (TMS) et de leurs facteurs de risque [12].

L'objectif de cette étude était d'estimer, à l'aide d'une modélisation, les facteurs prospectivement associés à la lombalgie chez les salariés des Pays de la Loire.

\section{MÉTHODES}

\section{Population et schéma de l'étude}

Cette étude transversale répétée reposait sur deux enquêtes menées successivement auprès d'une cohorte de salariés des Pays de la Loire.

La première phase de l'étude s'est déroulée entre 2002 et 2005. Quatre-vingt-trois médecins du travail volontaires ont effectué un tirage au sort parmi leurs salariés de 20 à 59 ans, vus pour leur visite périodique, employés d'une entreprise, privée ou publique, localisée dans la région, quel que soit le type de contrat de travail (CDI, CDD, intérim, etc.). Les taux de participation des médecins du travail par rapport aux effectifs régionaux étaient proches au sein des différents secteurs d'activité (18\% dans le secteur privé, $17 \%$ pour la fonction publique d'État, $15 \%$ pour la fonction publique hospitalière, et $14 \%$ pour le secteur agricole). Au total, 3710 sujets (2163 hommes et 1547 femmes) ayant donné leur consentement écrit ont ainsi été inclus aléatoirement et ont complété un auto-questionnaire. Ils représentaient $3,4 \%$ de l'ensemble des salariés des Pays de la Loire.

La deuxième phase de l'étude a débuté en février 2007, avec l'envoi d'un nouvel auto-questionnaire aux 3710 salariés précédemment inclus, suivi, en cas de 


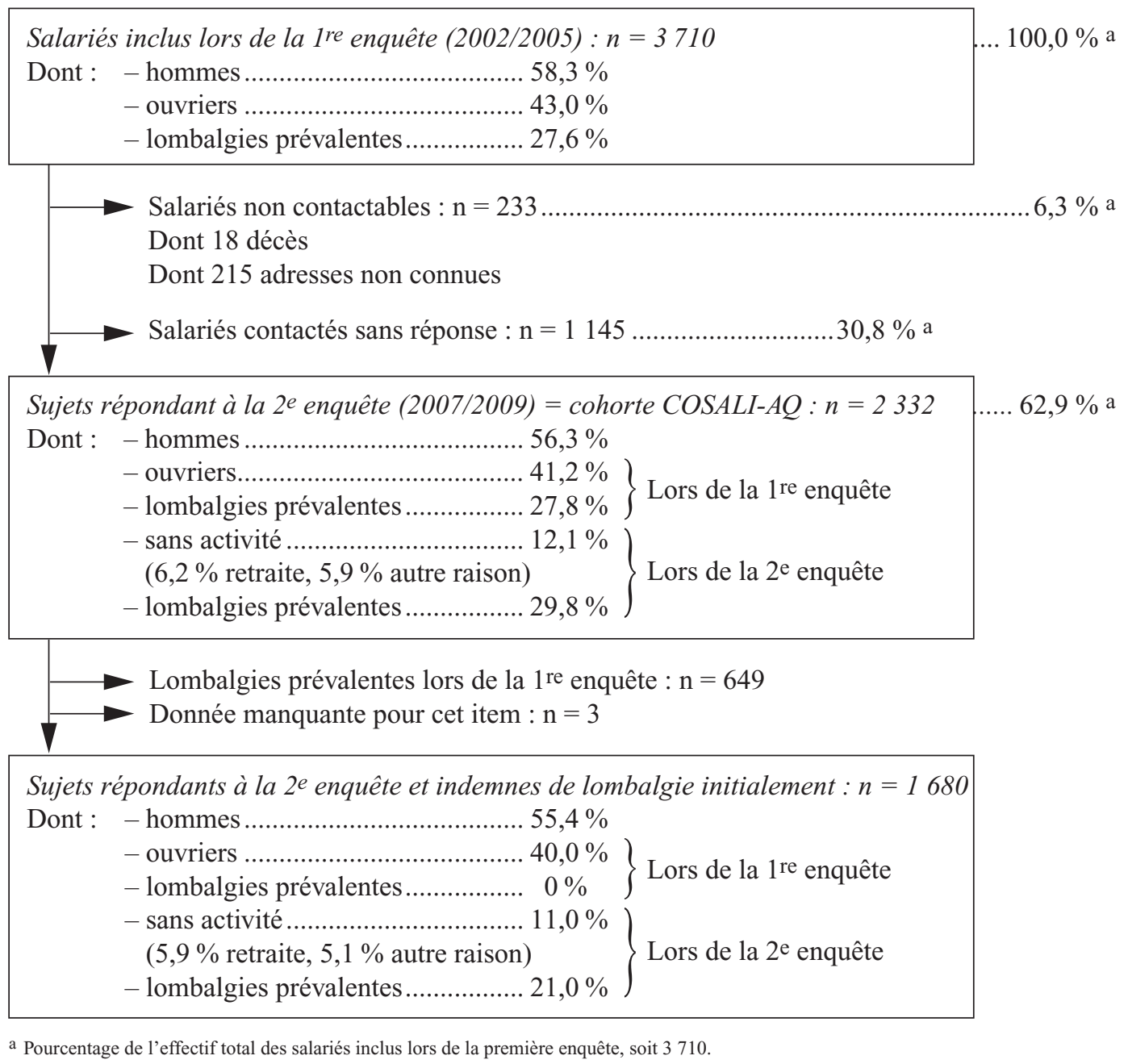

Figure 1. Diagramme de flux des participants à l'étude.

non-réponse, de courriers de relance, en juin puis septembre 2007. Parmi les 3710 sujets, 2332 (soit 62,9\%) ont répondu à la deuxième enquête entre 2007 et 2009, constituant la cohorte COSALI-AQ (COhorte des SAlariés LIgériens-Auto-Questionnaire) (figure 1). Il s'agissait de 1313 hommes et 1019 femmes (taux de réponse respectifs : $60,7 \%$ et $65,9 \%$ ).

Cette étude a fait l'objet d'une autorisation de la Commission nationale de l'informatique et des libertés (CNIL) en 2001 puis 2006.

\section{Critère d'intérêt principal}

Dans les deux enquêtes, un auto-questionnaire de type « nordique » [13] interrogeait le salarié sur l'existence de courbatures, gêne, douleurs, et/ou engourdissement du bas du dos (ci-après nommés "lombalgies ») au cours des sept jours précédant l'enquête. En admettant une prévalence de période d'une semaine, les lombalgies déclarées ont alors été considérées comme «prévalentes » au moment de l'enquête. Le critère d'intérêt principal de cette étude était la déclaration de lombalgies prévalentes lors de la deuxième enquête.

\section{Facteurs de risque et de confusion}

Ils reposaient sur les réponses à l'auto-questionnaire obtenues lors de la première enquête, et comprenaient :

\section{a) Des facteurs individuels}

- L'âge : en classes de dix ans.

- La grande taille : appartenance au quartile supérieur de la distribution parmi les sujets de la cohorte lors de la première enquête, pour chaque sexe (taille supérieure à $179 \mathrm{~cm}$ chez les hommes).

- Le surpoids ou l'obésité : indice de masse corporelle $(\mathrm{IMC}) \geq 25 \mathrm{~kg} / \mathrm{m}^{2}$, selon l'Organisation mondiale de la santé.

- Les douleurs du cou et/ou des membres supérieurs (MS) : symptômes au cours des sept jours précédant la première enquête dans au moins l'une des zones suivantes : nuque, épaule, bras, coude, avant-bras, poignet, main, doigts. 


\section{b) Des caractéristiques professionnelles}

- La catégorie socioprofessionnelle (CSP).

- Le type de contrat de travail : précaire (contrat de durée déterminée (CDD), intérim, stage, emploi aidé, apprentissage) / non précaire (contrat de durée indéterminée et titulaire de la fonction publique).

- L'ancienneté dans le poste.

\section{c) Des facteurs d'exposition professionnelle}

Ils ont été répartis en trois catégories :

Facteurs organisationnels

- Travailler plus que l'horaire officiellement prévu : oui/non.

- Connaître les tâches du jour en embauchant le matin : oui/non.

- Occuper différents postes au cours de son travail : oui/non.

- Avoir un salaire variable : oui/non.

- Travailler avec des collègues en situation précaire : oui/non.

- Avoir un rythme de travail imposé par : des délais de production/des contraintes industrielles (déplacement d'une pièce, cadence d'une machine)/les deux/aucune de ces contraintes.

- Contraintes marchandes (travail rythmé par une demande extérieure : public, clients...) : oui/non.

- Pouvoir s'interrompre ou changer de tâche pendant dix minutes chaque heure : oui/non.

\section{Facteurs biomécaniques}

- Se pencher plus de deux heures par jour : en avant/ sur le côté/les deux/aucun des deux.

- Cotation supérieure ou égale à 14 sur l'échelle de Borg (autoévaluation de l'intensité des efforts physiques produits au cours d'une journée de travail ; échelle étendue de 6 à 20).

- Conduire un engin de chantier, un tracteur ou un chariot automoteur au moins « rarement »: oui/non.

- Conduire un véhicule (automobile, camion, autobus) plus de quatre heures par jour: oui/non.

- Porter des charges de 10 à $25 \mathrm{~kg}$ au moins deux heures par jour et/ou des charges de plus de $25 \mathrm{~kg}$ au moins « rarement». En plus des modalités 'oui' et 'non', une troisième modalité 'donnée manquante' a été considérée car les données manquantes n'étaient pas distribuées aléatoirement. En effet, les non-répondants à cet item étaient plus fréquemment des hommes de plus de 50 ans, cadres ou professions intermédiaires, et/ou présentant des éléments en faveur d'une exposition variable dans le temps : ancienneté dans le poste inférieure à un an, contrat de travail précaire, différents postes occupés au cours de la journée. Les données manquantes pourraient donc être informatives et correspondre à une exposition intermédiaire (irrégulière ou difficilement chiffrable).
Facteurs psychosociaux

Le Job Content Questionnaire de Karasek est un questionnaire validé évaluant les contraintes psychosociales liées au travail selon cinq dimensions [14, 15]. L'exposition à chacune d'elles était définie par l'appartenance au quartile le plus péjoratif de la distribution lors de la première enquête, parmi les salariés de la cohorte, séparément pour chaque sexe (seuils donnés ici pour les hommes) : faible autonomie décisionnelle : score strictement inférieur à 32 ; faible utilisation des compétences : score $<32$; forte demande psychologique : score strictement supérieur à 24 ; faible soutien de la hiérarchie : score $<11$; faible soutien social des collègues : score $<12$.

\section{Analyses statistiques}

Ces analyses ont été menées indépendamment pour chaque sexe. Seuls les résultats concernant les hommes sont présentés ici.

\section{a) Statistiques descriptives}

La fréquence des lombalgies déclarées lors de la deuxième enquête a été décrite, globalement, puis en fonction des caractéristiques professionnelles initiales.

\section{b) Modélisation logistique multi-étapes du risque de lombalgie}

Un processus de régression logistique binaire multiétapes a été utilisé pour modéliser le risque de lombalgies lors de la deuxième enquête par les facteurs de risque recueillis lors de la première enquête. Les effets potentiels de ces facteurs ont donc été estimés par des odds-ratios $(\mathrm{OR})$ et leurs intervalles de confiance à $95 \%$ (IC $95 \%$ ).

Des liens très forts existent entre les caractéristiques professionnelles (CSP, contrat de travail, ancienneté) d'une part, et les facteurs individuels et d'exposition professionnelle d'autre part (en particulier ancienneté et âge, CSP et facteurs biomécaniques [16]). C'est pourquoi seuls les facteurs individuels et d'exposition professionnelle ont été considérés comme facteurs de risque potentiels.

Les différentes étapes du processus de modélisation, inspiré de la méthode décrite par Hosmer et Lemeshow [17], ont été les suivantes :

- Étape 1 : Une régression logistique univariée a estimé le lien brut entre chaque facteur de risque et le critère d'intérêt principal.

- Étape 2 : À l'intérieur de chacun des quatre groupes de facteurs de risque (individuels, organisationnels, biomécaniques, et psychosociaux), les variables liées au critère d'intérêt principal en analyse univariée avec une $\mathrm{p}$-value $(\mathrm{p})$ inférieure à 0,20 ont été 
Tableau I

Distribution des 3710 salariés inclus lors de la $1^{\text {re }}$ enquête par sexe, âge et catégorie socioprofessionnelle (CSP), comparativement aux données du recensement INSEE 1999 pour la région Pays de la Loire.

\begin{tabular}{|c|c|c|c|c|c|}
\hline & \multirow[b]{2}{*}{ Caractéristiques } & \multirow{2}{*}{$\begin{array}{c}\text { Salariés inclus } \\
\text { lors de la } 1^{\text {re }} \text { enquête : } \\
n(\%)\end{array}$} & \multicolumn{2}{|c|}{ Données régionales INSEE 1999} & \multirow[b]{2}{*}{$\mathrm{p}\left(\mathrm{Chi}^{2}\right)$} \\
\hline & & & Population régionale de référence & $\begin{array}{l}\text { Distribution régionale : } \\
\qquad \mathrm{n}(\%)\end{array}$ & \\
\hline \multirow{3}{*}{ Sexe } & Masculin & $2163(58,3)$ & & $576469(53,1)$ & \\
\hline & Féminin & $1547(41,7)$ & & $508186(46,9)$ & $<0,0001$ \\
\hline & Total & $3710(100,0)$ & Salariés 20/59 ans & $1084655(100,0)$ & \\
\hline \multirow{5}{*}{ Âge } & 20 à 29 ans & $841(22,7)$ & & $260004(20,7)$ & \\
\hline & 30 à 39 ans & $1085(29,2)$ & & $370653(29,6)$ & \\
\hline & 40 à 49 ans & $1095(29,5)$ & & $385297(30,7)$ & 0,027 \\
\hline & 50 à 59 ans & $689(18,6)$ & & $238347(19,0)$ & \\
\hline & Total & $3710(100,0)$ & Actifs occupés $20 / 59$ ans & $1254301(100,0)$ & \\
\hline \multirow{5}{*}{ CSP } & Ouvrier & $1586(43,0)$ & & $403195(35,4)$ & \\
\hline & Employé & $986(26,7)$ & & $348761(30,7)$ & \\
\hline & $\begin{array}{l}\text { Profession } \\
\text { intermédiaire }\end{array}$ & $829(22,5)$ & & $268039(23,6)$ & $<0,0001$ \\
\hline & Cadre & $288(7,8)$ & & $117012(10,3)$ & \\
\hline & Total $^{\mathrm{b}}$ & $3689(100,0)$ & $\begin{array}{r}\text { Actifs occupés 20/59 ans } \\
\text { hors artisans et exploitants agricoles }\end{array}$ & $1137007(100,0)$ & \\
\hline
\end{tabular}

a INSEE :Institut national de la statistique et des études économiques.

${ }^{b}$ Les sujets avec donnée manquante pour la catégorie socioprofessionnelle ont été exclus des analyses.

entrées dans un modèle logistique multivarié par groupe de variables, dit « sous-modèle », puis sélectionnées selon une procédure manuelle descendante, pour ne conserver que les variables liées au critère d'intérêt principal $(p<0,10)$, et les facteurs de confusion (ceux dont la suppression modifiait l'estimation d'au moins un paramètre d'intérêt de plus de $10 \%$ ).

- Étape 3 : Toutes les variables retenues à l'étape 2 ont été introduites dans un modèle multivarié global, puis sélectionnées selon une procédure de sélection similaire à celle de l'étape 2 , pour ne conserver que les variables liées au critère d'intérêt principal $(\mathrm{p}<0,05)$ et les facteurs de confusion.

Cette stratégie multi-étapes a été choisie afin de limiter le nombre de variables candidates dans le modèle final, et de réduire les phénomènes de multi colinéarité.

Des analyses de sensibilité ont été menées à partir de cette analyse principale. D'une part, l'ensemble de la stratégie de modélisation a été réitérée en retirant de la liste des variables candidates le facteur 'douleurs du cou et/ou des MS', pour éviter le potentiel surajustement des modèles comportant ce facteur. D'autre part, les analyses ont été menées de nouveau en restreignant la population d'étude aux sujets indemnes de lombalgie initialement, définis par l'absence de lombalgie prévalente lors de la première enquête, afin de s'affranchir des lombalgies chroniques ou récurrentes depuis la première enquête.
Les observations présentant des données manquantes pour certaines variables ont été exclues des modèles comportant ces variables, sauf pour la variable 'porter des charges', considérée en trois modalités : 'exposé', 'non exposé, 'donnée manquante'.

Les analyses ont été réalisées avec le logiciel SAS version 9.2 (SAS Institute, Cary, NC). Sauf indication contraire, le seuil retenu pour la significativité statistique était 0,05 .

\section{RÉSULTATS}

\section{Représentativité de la population d'étude}

L'échantillon des 3710 salariés inclus lors de la première enquête comportait, en proportion, plus d'hommes que la population salariée des Pays de la Loire de 20 à 59 ans, telle que déterminée par le recensement de l'Institut national de la statistique et des études économiques (INSEE) de 1999 (58,3\% versus 53,1\%, $\mathrm{p}<0,0001)$. Comparativement à la population active occupée de la région (sans distinction possible entre salariés et non-salariés), l'échantillon comportait plus de jeunes de 20 à 29 ans $(22,7 \%$ versus $20,7 \%$, $\mathrm{p}=0,027)$, et plus d'ouvriers $(43,0 \%$ versus $35,4 \%$, $\mathrm{p}<0,0001$ ) (tableau I).

À l'opposé des résultats précédents, les répondants à la deuxième enquête, constituant la cohorte COSALI-AQ, 
Tableau II

Distribution comparative des répondants et des non-répondants à la deuxième enquête par sexe, âge et catégorie socioprofessionnelle (CSP).

\begin{tabular}{|c|c|c|c|c|}
\hline & Caractéristiques & $\begin{array}{c}\text { Répondants }(\mathrm{n}=2332): \\
\mathrm{n}(\%)\end{array}$ & $\begin{array}{c}\text { Non-répondants }(\mathrm{n}=1378) \text { : } \\
\mathrm{n}(\%)\end{array}$ & $\mathrm{p}\left(\mathrm{Chi}^{2}\right)$ \\
\hline \multirow{3}{*}{ Sexe } & Masculin & $1313(56,3)$ & $850(61,7)$ & \\
\hline & Féminin & $1019(43,7)$ & $528(38,3)$ & 0,0015 \\
\hline & Total & $2332(100,0)$ & $1378(100,0)$ & \\
\hline \multirow{5}{*}{ Âge } & 20 à 29 ans & $413(17,7)$ & $428(31,1)$ & \\
\hline & 30 à 39 ans & $677(29,0)$ & $408(29,6)$ & \\
\hline & 40 à 49 ans & $758(32,5)$ & $337(24,4)$ & $<0,0001$ \\
\hline & 50 à 59 ans & $484(20,8)$ & $205(14,9)$ & \\
\hline & Total & $2332(100,0)$ & $1378(100,0)$ & \\
\hline \multirow{5}{*}{ CSP } & Ouvrier & $960(41,2)$ & $626(45,9)$ & \\
\hline & Employé & $616(26,5)$ & $370(27,1)$ & \\
\hline & Profession intermédiaire & $553(23,8)$ & $276(20,2)$ & 0,0078 \\
\hline & Cadre & $195(8,4)$ & $93(6,8)$ & \\
\hline & Total ${ }^{\mathrm{a}}$ & $2324(100,0)$ & $1365(100,0)$ & \\
\hline
\end{tabular}

${ }^{a}$ Les sujets avec donnée manquante pour la catégorie socioprofessionnelle ont été exclus des analyses.

Tableau III

Comparaison des caractéristiques professionnelles lors de la première enquête

selon la présence ou l'absence de lombalgie lors de la deuxième enquête chez les hommes de la cohorte COSALI-AQ.

\begin{tabular}{|c|c|c|c|c|}
\hline \multicolumn{2}{|c|}{ Caractéristiques professionnelles } & $\begin{array}{c}\text { Lombalgie }(\mathrm{n}=394): \\
\mathrm{n}(\%)\end{array}$ & $\begin{array}{c}\text { Absence de lombalgie }(\mathrm{n}=910) \text { : } \\
\mathrm{n}(\%)\end{array}$ & $\mathrm{p}^{\mathrm{a}}$ \\
\hline \multirow{5}{*}{$\begin{array}{l}\text { Catégorie socio- } \\
\text { professionnelle }\end{array}$} & Ouvrier & $233(59,1)$ & $467(51,3)$ & \multirow{5}{*}{$\mathbf{0 , 0 0 1 3}$} \\
\hline & Employé & $30(7,6)$ & $73(8,0)$ & \\
\hline & Profession intermédiaire & $106(26,9)$ & $247(27,1)$ & \\
\hline & Cadre & $25(6,4)$ & $123(13,5)$ & \\
\hline & Total & $394(100,0)$ & $910(100,0)$ & \\
\hline \multirow{3}{*}{ Contrat $^{b}$} & Non précaire & $365(92,6)$ & $840(92,6)$ & \multirow{3}{*}{0,99} \\
\hline & Précaire & $29(7,4)$ & $67(7,4)$ & \\
\hline & Total $^{\mathrm{d}}$ & $394(100,0)$ & $907(100,0)$ & \\
\hline \multirow{5}{*}{$\begin{array}{l}\text { Ancienneté } \\
\text { dans le poste }\end{array}$} & $<1$ an & $37(9,5)$ & $88(9,8)$ & \multirow{5}{*}{$0,71^{\mathrm{c}}$} \\
\hline & 1 à 2 ans & $59(15,1)$ & $140(15,6)$ & \\
\hline & 3 à 10 ans & $138(35,3)$ & $288(32,1)$ & \\
\hline & $>10$ ans & $157(40,1)$ & $382(42,5)$ & \\
\hline & Total $^{\mathrm{d}}$ & $391(100,0)$ & $898(100,0)$ & \\
\hline
\end{tabular}

Lombalgie $=$ salariés déclarant des lombalgies dans les sept jours précédant la deuxième enquête.

Abence de lombalgie = salariés ne déclarant pas de lombalgie dans les sept jours précédant la deuxième enquête.

a Comparaison entre lombalgiques et non-lombalgiques - sauf indication contraire, le test utilisé est le test du Chi ${ }^{2}$.

b Type de contrat (non précaire : fonctionnaire et CDI ; précaire : CDD, intérimaire, stagiaire, emploi aidé, apprenti)

c Test non paramétrique de Mann-Whitney-Wilcoxon pour données ordonnées.

${ }^{\mathrm{d}}$ Les sujets avec donnée manquante pour cet item ont été exclus des analyses.

étaient, en proportion, moins souvent des hommes, des jeunes, et des ouvriers, que les non-répondants (tableau II).

\section{Statistiques descriptives}

Dans la cohorte COSALI-AQ, 394 hommes (30,0\%) déclaraient avoir eu des lombalgies dans les sept jours précédant la deuxième enquête. La CSP, relevée lors de la première enquête, différait significativement entre les lombalgiques et les non-lombalgiques lors de la deuxième enquête. Les pourcentages respectifs de cadres et d'ouvriers étaient ainsi de $6,4 \%$ et $59,1 \%$ chez les lombalgiques, contre $13,5 \%$ et $51,3 \%$ chez les non-lombalgiques $(\mathrm{p}=0,0013)$. En revanche, la répartition selon le type de contrat ou l'ancienneté ne différait pas significativement (tableau III). 
Tableau IV

Liaisons univariées entre chaque facteur de risque et la fréquence des lombalgies déclarées lors de la deuxième enquête par les hommes de la cohorte COSALI-AQ.

\begin{tabular}{|c|c|c|c|c|c|}
\hline & Facteurs de risque lors de la prem & ière enquête & $\mathrm{n} / \mathrm{N}_{\exp }{ }^{\mathrm{a}}$ & OR $(\mathrm{IC} 95 \%)^{\mathrm{b}}$ & $\mathrm{p}^{\mathrm{b}}$ \\
\hline \multirow{4}{*}{ Individuels } & Âge $\left(\right.$ classes de 10 ans) ${ }^{\mathrm{c}}$ & & - & - & 0,21 \\
\hline & Grande taille $(>179 \mathrm{~cm})$ & & $99 / 324$ & $1,03(0,78 ; 1,35)$ & 0,85 \\
\hline & Surpoids/obésité (IMC $\geq 25$ kg/m²) & & $186 / 579$ & $1,18(0,93 ; 1,50)$ & 0,18 \\
\hline & Douleurs cou/membre supérieur & & $207 / 465$ & $2,79(2,18 ; 3,57)$ & $<0,0001$ \\
\hline \multirow{11}{*}{ Organisationnels } & Travailler plus que prévu & & $270 / 858$ & $1,25(0,96 ; 1,61)$ & 0,092 \\
\hline & Ne pas connaître les tâches du jour & & $58 / 171$ & $1,21(0,86 ; 1,70)$ & 0,27 \\
\hline & Occuper différents postes & & $144 / 479$ & $0,97(0,76 ; 1,25)$ & 0,82 \\
\hline & Avoir un salaire variable & & $105 / 300$ & $1,34(1,02 ; 1,76)$ & $\mathbf{0 , 0 3 9}$ \\
\hline & $\begin{array}{l}\text { Travailler avec des collègues } \\
\text { en situation précaire }\end{array}$ & & $113 / 371$ & $1,02(0,78 ; 1,32)$ & 0,90 \\
\hline & \multirow{4}{*}{ Rythme } & Pas de contrainte (référence) & $147 / 557$ & 1,00 & \multirow{4}{*}{0,0078} \\
\hline & & Délais de production & $158 / 507$ & $1,26(0,97 ; 1,65)$ & \\
\hline & & Contrainte industrielle & $16 / 54$ & $1,17(0,64 ; 2,17)$ & \\
\hline & & Les deux & $64 / 158$ & $1,90(1,31 ; 2,75)$ & \\
\hline & Contraintes marchandes & & $175 / 583$ & $1,00(0,78 ; 1,27)$ & 0,97 \\
\hline & Ne pas pouvoir s'interrompre & & $134 / 428$ & $1,08(0,84 ; 1,39)$ & 0,55 \\
\hline \multirow{10}{*}{ Biomécaniques } & \multirow{4}{*}{ Se pencher } & Ne pas se pencher (référence) & $206 / 811$ & 1,00 & \multirow{4}{*}{$<0,0001$} \\
\hline & & Se pencher en avant $>2 \mathrm{~h} / \mathrm{j}$ & $98 / 277$ & $1,61(1,20 ; 2,16)$ & \\
\hline & & Se pencher sur le côté $>2 \mathrm{~h} / \mathrm{j}$ & $10 / 23$ & $2,26(0,98 ; 5,23)$ & \\
\hline & & Les deux $>2 \mathrm{~h} / \mathrm{j}$ & $78 / 187$ & $2,10(1,51 ; 2,93)$ & \\
\hline & Échelle de Borg $\geq 14$ & & $132 / 390$ & $1,28(0,99 ; 1,65)$ & 0,060 \\
\hline & Conduire un engin & & $100 / 277$ & $1,41(1,06 ; 1,86)$ & $\mathbf{0 , 0 1 7}$ \\
\hline & Conduire un véhicule $>4 \mathrm{~h} / \mathrm{j}$ & & $50 / 143$ & $1,28(0,89 ; 1,85)$ & 0,19 \\
\hline & \multirow{3}{*}{$\begin{array}{l}\text { Porter des charges }>25 \mathrm{~kg} \\
\geq \text { rarement } \\
\text { ou } 10-25 \mathrm{~kg}>2 \mathrm{~h} / \mathrm{j}\end{array}$} & Non (référence) & $213 / 769$ & 1,00 & \multirow{3}{*}{0,060} \\
\hline & & Oui & $123 / 361$ & $1,35(1,03 ; 1,77)$ & \\
\hline & & Donnée manquante & $58 / 174$ & $1,31(0,92 ; 1,86)$ & \\
\hline \multirow{5}{*}{ Psychosociaux } & \multicolumn{2}{|l|}{ Faible autonomie décisionnelle ${ }^{\mathrm{d}}$} & $53 / 166$ & $1,09(0,77 ; 1,55)$ & 0,63 \\
\hline & \multicolumn{2}{|l|}{ Faible utilisation des compétences ${ }^{d}$} & $108 / 303$ & $1,38(1,05 ; 1,81)$ & 0,022 \\
\hline & \multicolumn{2}{|l|}{ Forte demande psychologique ${ }^{\mathrm{d}}$} & $73 / 263$ & $0,86(0,64 ; 1,16)$ & 0,32 \\
\hline & \multicolumn{2}{|l|}{ Faible soutien de la hiérarchie ${ }^{\mathrm{d}}$} & $113 / 308$ & $1,48(1,13 ; 1,94)$ & 0,0043 \\
\hline & \multicolumn{2}{|l|}{ Faible soutien des collègues ${ }^{\mathrm{d}}$} & $84 / 242$ & $1,30(0,96 ; 1,74)$ & 0,087 \\
\hline
\end{tabular}

\section{Modélisation logistique multi-étapes du risque de lombalgie}

\section{a) Analyse univariée}

Des douleurs initiales du cou et/ou des MS étaient le facteur le plus fortement associé à la présence de lombalgie lors de la deuxième enquête chez les hommes de la cohorte COSALI-AQ. Un salaire variable, des contraintes de rythme du poste de travail, se pencher plus de deux heures par jour, la conduite d'engins, une faible utilisation des compétences et un faible soutien de la hiérarchie étaient également significativement liés en univarié avec les lombalgies prévalentes ultérieures $(\mathrm{p}<0,05)$ (tableau IV).

\section{b) Modélisation multivariée du risque de lombalgie chez les hommes de la cohorte COSALI-AQ}

Le modèle multivarié final comportait quatre variables significatives. La présence initiale de douleurs du cou et/ou des MS était le facteur le plus lié à la présence de lombalgie ultérieure, avec un OR à 2,73 ( $\mathrm{p}<0,0001)$. 'Travailler plus que prévu', était modérément lié au critère d'intérêt principal $(\mathrm{OR}=1,46 ; \mathrm{p}=0,0064)$. Enfin, deux facteurs biomécaniques y étaient significativement associés : la conduite d'engins $(\mathrm{OR}=1,40$; $\mathrm{p}=0,03)$, et surtout 'se pencher plus de deux heures par jour' 'en avant' ou 'en avant et sur le côté', avec des OR respectifs à 1,56 et $1,84(\mathrm{p}=0,0013)$ (tableau V). 
Tableau V

Modélisation logistique binaire du risque de lombalgie dans la deuxième enquête chez les hommes de la cohorte COSALI-AQ (quatre sous-modèles multivariés par groupe de variables et modèle multivarié final, réduits).

\begin{tabular}{|c|c|c|c|c|c|c|c|c|}
\hline \multirow{2}{*}{\multicolumn{3}{|c|}{ Facteurs de risque lors de la première enquête }} & \multicolumn{3}{|c|}{ Quatre sous-modèles réduits ${ }^{\mathrm{a}}$} & \multicolumn{3}{|c|}{ Modèle multivarié final $(\mathrm{n}=1265)$} \\
\hline & & & $\mathrm{n} / \mathrm{N}_{\exp }{ }^{\mathrm{b}}$ & OR $(\mathrm{IC} 95 \%)^{\mathrm{c}}$ & $\mathrm{p}^{\mathrm{c}}$ & $\mathrm{n} / \mathrm{N}_{\exp }{ }^{\mathrm{b}}$ & OR $(\mathrm{IC} 95 \%)^{\mathrm{c}}$ & $\mathrm{p}^{\mathrm{c}}$ \\
\hline Ind $^{\mathrm{d}}$ & \multicolumn{2}{|c|}{ Douleurs cou/membre supérieur } & $207 / 465$ & $2,79(2,18 ; 3,57)$ & $<0,0001$ & $203 / 458$ & $2,73(2,12 ; 3,52)$ & $<0,0001$ \\
\hline \multirow{6}{*}{ Organisationnels } & \multicolumn{2}{|c|}{ Travailler plus que prévu } & $262 / 827$ & $1,26(0,96 ; 1,65)$ & 0,096 & $267 / 844$ & $1,46(1,11 ; 1,92)$ & 0,0064 \\
\hline & \multicolumn{2}{|c|}{ Avoir un salaire variable } & $102 / 290$ & $1,32(1,00 ; 1,75)$ & 0,054 & - & - & - \\
\hline & \multirow{4}{*}{ Rythme } & Pas de contrainte (référence) & $145 / 542$ & 1,00 & 0,022 & - & - & - \\
\hline & & Délais de production & $155 / 496$ & $1,24(0,94 ; 1,62)$ & & - & - & \\
\hline & & Contrainte industrielle & $14 / 50$ & $1,09(0,57 ; 2,09)$ & & - & - & \\
\hline & & Les deux & $59 / 151$ & $1,81(1,24 ; 2,65)$ & & - & - & \\
\hline \multirow{5}{*}{ Biomécaniques } & \multirow{4}{*}{$\begin{array}{l}\text { Se } \\
\text { pencher }\end{array}$} & Ne pas se pencher (référence) & $206 / 808$ & 1,00 & $<0,0001$ & $200 / 794$ & 1,00 & \multirow{4}{*}{0,0013} \\
\hline & & Se pencher en avant $>2 \mathrm{~h} / \mathrm{j}$ & $98 / 277$ & $1,54(1,14 ; 2,07)$ & & $97 / 271$ & $1,56(1,14 ; 2,12)$ & \\
\hline & & Se pencher sur le côté $>2 \mathrm{~h} / \mathrm{j}$ & $10 / 23$ & $2,12(0,91 ; 4,93)$ & & $9 / 22$ & $1,62(0,66 ; 3,95)$ & \\
\hline & & Les deux $>2 \mathrm{~h} / \mathrm{j}$ & $78 / 187$ & $2,05(1,47 ; 2,85)$ & & $75 / 178$ & $1,84(1,29 ; 2,62)$ & \\
\hline & \multicolumn{2}{|c|}{ Conduire un engin } & $100 / 275$ & $1,30(0,98 ; 1,73)$ & 0,074 & $98 / 268$ & $1,40(1,03 ; 1,89)$ & $\mathbf{0 , 0 3 0}$ \\
\hline \multirow{2}{*}{$\operatorname{PS}^{\mathrm{d}}$} & \multicolumn{2}{|c|}{ Faible utilisation des compétences $\mathrm{e}^{\mathrm{e}}$} & $106 / 297$ & $1,31(0,99 ; 1,73)$ & 0,062 & - & - & - \\
\hline & \multicolumn{2}{|c|}{ Faible soutien de la hiérarchie $\mathrm{e}^{\mathrm{e}}$} & $113 / 307$ & $1,42(1,08 ; 1,87)$ & 0,012 & - & - & - \\
\hline \multicolumn{9}{|c|}{ 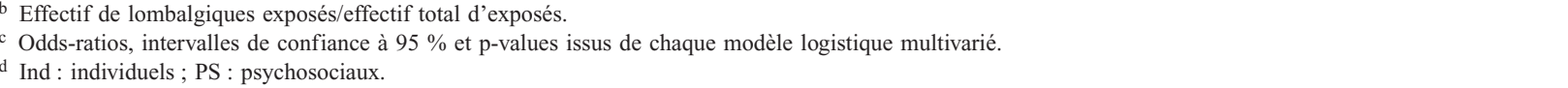 } \\
\hline
\end{tabular}

\section{c) Analyses de sensibilité}

\section{Modélisation du risque de lombalgie sans le facteur 'douleurs du cou et/ou des MS'}

Les analyses précédentes ont été réitérées dans leur intégralité après avoir retiré de la liste des variables candidates le facteur individuel 'douleurs du cou et/ou des MS'. Comparativement au modèle précédent, un nouveau facteur était mis en évidence : un faible soutien de la hiérarchie était modérément mais significativement associé au risque de lombalgie ultérieure $(\mathrm{OR}=1,35 ; \mathrm{p}=0,038)$. Par ailleurs, l'estimation $\mathrm{du}$ risque lié à 'se pencher plus de deux heures par jour en avant et sur le côté' était augmentée, avec un OR passant de 1,84, dans l'analyse principale, à 2,13 dans ce modèle $(\mathrm{p}=0,0001)$. Les autres effets estimés, concernant les facteurs 'travailler plus que prévu', et 'conduire un engin', étaient peu modifiés (tableau VI).

\section{Modélisation du risque de lombalgie chez les hommes indemnes de lombalgie initialement}

Parmi les 1313 hommes de la cohorte COSALI-AQ, 931 ne déclaraient pas de lombalgie prévalente lors de la première enquête et ont constitué le sous-groupe « indemne de lombalgie initialement ». Parmi eux, la prévalence des lombalgies lors de la deuxième enquête était de $21,2 \%$ (figure 1 ). Après avoir réitéré l'intégralité des analyses chez ce sous-groupe, trois variables, déjà mises en évidence dans l'analyse principale, étaient significativement associées au risque ultérieur de lombalgie dans le modèle multivarié final: 'douleurs du cou et/ou des MS', 'travailler plus que prévu', et 'se pencher plus de $2 \mathrm{~h}$ par jour'. Comparativement à l'analyse principale, l'effet du facteur 'douleurs du cou et/ou des MS' était assez stable, alors que ceux des facteurs 'travailler plus que prévu' et 'se pencher' (notamment 'en avant et sur le côté') étaient accrus, avec des OR majorés de $30 \%$ environ. Dans ce sous-groupe, la conduite d'engins n'était plus significativement liée au risque de lombalgie (tableau VII).

\section{DISCUSSION}

Notre population d'étude était globalement représentative de celle des salariés des Pays de la Loire. L'échantillon des 3710 sujets inclus lors de la première enquête comportait plus d'hommes, d'ouvriers, et de jeunes que la population régionale cible. En revanche, ces trois catégories étaient celles ayant moins participé à la deuxième enquête, ce qui est assez fréquemment retrouvé dans la littérature [18]. Malgré des différences modérées, mais néanmoins statistiquement significatives, entre notre population d'étude et la population régionale, des calages sur marge ont permis de constater que les fréquences d'exposition observées chez les salariés étaient peu modifiées par un redressement en fonction du sexe, de l'âge, et de la CSP (données non présentées). Le taux de participation à la deuxième enquête parmi l'échantillon initial (62,9\%) était relati- 
Tableau VI

Modélisation logistique binaire du risque de lombalgie dans la deuxième enquête chez les hommes de la cohorte COSALI-AQ, après suppression du facteur " douleurs du cou et/ou des MS »

(trois sous-modèles multivariés par groupe de variables et modèle multivarié final, réduits).

\begin{tabular}{|c|c|c|c|c|c|c|c|c|}
\hline \multirow{2}{*}{\multicolumn{3}{|c|}{ Facteurs de risque lors de la première enquête }} & \multicolumn{3}{|c|}{ Trois sous-modèles réduits ${ }^{a}$} & \multicolumn{3}{|c|}{ Modèle multivarié final $(\mathrm{n}=1255)$} \\
\hline & & & $\mathrm{n} / \mathrm{N}_{\exp }^{\mathrm{b}}$ & OR $(\text { IC } 95 \%)^{\mathrm{c}}$ & $\mathrm{p}^{\mathrm{c}}$ & $\mathrm{n} / \mathrm{N}_{\exp }{ }^{\mathrm{b}}$ & OR (IC $95 \%)^{\mathrm{c}}$ & $\mathrm{p}^{\mathrm{c}}$ \\
\hline \multirow{6}{*}{ Organisationnels } & \multirow{2}{*}{\multicolumn{2}{|c|}{$\begin{array}{l}\text { Travailler plus que prévu } \\
\text { Avoir un salaire variable }\end{array}$}} & $262 / 827$ & $1,26(0,96 ; 1,65)$ & 0,096 & $264 / 842$ & $1,38(1,05 ; 1,81)$ & 0,021 \\
\hline & & & $102 / 290$ & $1,32(1,00 ; 1,75)$ & 0,054 & - & - & - \\
\hline & \multirow{4}{*}{ Rythme } & Pas de contrainte (référence) & $145 / 542$ & 1,00 & 0,022 & - & - & - \\
\hline & & Délais de production & $155 / 496$ & $1,24(0,94 ; 1,62)$ & & - & - & \\
\hline & & Contrainte industrielle & $14 / 50$ & $1,09(0,57 ; 2,09)$ & & - & - & \\
\hline & & Les deux & $59 / 151$ & $1,81(1,24 ; 2,65)$ & & - & - & \\
\hline \multirow{5}{*}{ Biomécaniques } & \multirow{4}{*}{$\begin{array}{l}\text { Se } \\
\text { pencher }\end{array}$} & Ne pas se pencher (référence) & $206 / 808$ & 1,00 & $<0,0001$ & $199 / 785$ & 1,00 & \multirow{4}{*}{0,0001} \\
\hline & & Se pencher en avant $>2 \mathrm{~h} / \mathrm{j}$ & $98 / 277$ & $1,54(1,14 ; 2,07)$ & & $92 / 269$ & $1,45(1,07 ; 1,97)$ & \\
\hline & & Se pencher sur le côté $>2 \mathrm{~h} / \mathrm{j}$ & $10 / 23$ & $2,12(0,91 ; 4,93)$ & & $9 / 22$ & $1,78(0,74 ; 4,27)$ & \\
\hline & & Les deux $>2 \mathrm{~h} / \mathrm{j}$ & $78 / 187$ & $2,05(1,47 ; 2,85)$ & & $76 / 179$ & $2,13(1,52 ; 3,00)$ & \\
\hline & \multicolumn{2}{|c|}{ Conduire un engin $\geq$ rarement } & $100 / 275$ & $1,30(0,98 ; 1,73)$ & 0,074 & $92 / 267$ & $1,35(1,00 ; 1,81)$ & 0,047 \\
\hline \multirow{2}{*}{$\operatorname{PS}^{\mathrm{d}}$} & \multicolumn{2}{|c|}{ Faible utilisation des compétences ${ }^{\mathrm{e}}$} & $106 / 297$ & $1,31(0,99 ; 1,73)$ & 0,062 & $101 / 289$ & $1,28(0,95 ; 1,72)$ & $0,11^{\mathrm{f}}$ \\
\hline & \multicolumn{2}{|c|}{ Faible soutien de la hiérarchie ${ }^{\mathrm{e}}$} & $113 / 307$ & $1,42(1,08 ; 1,87)$ & 0,012 & $108 / 300$ & $1,35(1,02 ; 1,79)$ & $\mathbf{0 , 0 3 8}$ \\
\hline \multicolumn{9}{|c|}{$\begin{array}{l}\text { a Effectifs utilisés pour chacun des sous-modèles, dans l'ordre de présentation : } 1239 ; 1295 ; 1277 . \\
\text { b Effectif de lombalgiques exposés/effectif total d'exposés. } \\
\text { c Odds-ratios, intervalles de confiance à } 95 \% \text { et p-values issus de chaque modèle logistique multivarié. } \\
\text { d PS : psychosociaux. } \\
\text { e Exposition définie par l'appartenance au quartile le plus péjoratif chez les hommes de la cohorte COSALI-AQ. } \\
\text { f Facteur non significatif mais agissant comme facteur de confusion. }\end{array}$} \\
\hline
\end{tabular}

Tableau VII

Modélisation logistique binaire du risque de lombalgie dans la deuxième enquête chez les hommes indemnes de lombalgie initialement (trois sous-modèles multivariés par groupe de variables et modèle multivarié final, réduits).

\begin{tabular}{|c|c|c|c|c|c|c|c|c|}
\hline \multirow{2}{*}{\multicolumn{3}{|c|}{ Facteurs de risque lors de la première enquête }} & \multicolumn{3}{|c|}{ Trois sous-modèles réduits ${ }^{\mathrm{a}}$} & \multicolumn{3}{|c|}{ Modèle multivarié final $(\mathrm{n}=898)$} \\
\hline & & & $\mathrm{n} / \mathrm{N}_{\exp }{ }^{\mathrm{b}}$ & OR $(\operatorname{IC~} 95 \%)^{\mathrm{c}}$ & $\mathrm{p}^{\mathrm{c}}$ & $\mathrm{n} / \mathrm{N}_{\exp }{ }^{\mathrm{b}}$ & OR $(\text { IC } 95 \%)^{\mathrm{c}}$ & $\mathrm{p}^{\mathrm{c}}$ \\
\hline Ind $^{\mathrm{d}}$ & \multicolumn{2}{|c|}{ Douleurs cou/membre supérieur } & $85 / 253$ & $2,53(1,82 ; 3,53)$ & $<0,0001$ & $84 / 250$ & $2,64(1,87 ; 3,72)$ & $<0,0001$ \\
\hline \multirow{7}{*}{ Organisationnels } & \multicolumn{2}{|c|}{ Travailler plus que prévu } & $129 / 550$ & $1,62(1,09 ; 2,39)$ & 0,016 & $140 / 598$ & $1,87(1,28 ; 2,73)$ & 0,0012 \\
\hline & \multicolumn{2}{|c|}{ Occuper différents postes $\geq 1 /$ semaine } & $54 / 305$ & $0,69(0,48 ; 0,99)$ & 0,045 & - & - & - \\
\hline & \multicolumn{2}{|c|}{ Avoir un salaire variable } & $47 / 186$ & $1,41(0,95 ; 2,08)$ & 0,088 & - & - & - \\
\hline & \multirow{4}{*}{ Rythme } & Pas de contrainte (référence) & $60 / 357$ & 1,00 & - & - & - & - \\
\hline & & Délais de production & $79 / 340$ & $1,44(0,99 ; 2,11)$ & & - & - & \\
\hline & & Contrainte industrielle & $5 / 31$ & $0,93(0,34 ; 2,54)$ & & - & - & \\
\hline & & Les deux & 28/99 & $2,11(1,25 ; 3,56)$ & & - & - & \\
\hline \multirow{4}{*}{ Biomécaniques } & \multirow{4}{*}{$\begin{array}{l}\text { Se } \\
\text { pencher }\end{array}$} & Ne pas se pencher (référence) & $105 / 608$ & 1,00 & \multirow{4}{*}{0,0001} & $102 / 600$ & 1,00 & \multirow{4}{*}{0,0005} \\
\hline & & Se pencher en avant $>2 \mathrm{~h} / \mathrm{j}$ & $45 / 182$ & $1,57(1,06 ; 2,34)$ & & $44 / 176$ & $1,69(1,12 ; 2,56)$ & \\
\hline & & Se pencher sur le côté $>2 \mathrm{~h} / \mathrm{j}$ & $5 / 15$ & $2,40(0,80 ; 7,15)$ & & $4 / 14$ & $1,78(0,54 ; 5,93)$ & \\
\hline & & Les deux $>2 \mathrm{~h} / \mathrm{j}$ & $40 / 115$ & $2,56(1,65 ; 3,96)$ & & $38 / 108$ & $2,51(1,58 ; 3,99)$ & \\
\hline
\end{tabular}

a Effectifs utilisés pour chacun des sous-modèles, dans l'ordre de présentation : $914 ; 827 ; 920$.

b Effectif de lombalgiques exposés/effectif total d'exposés.

c Odds-ratios, intervalles de confiance à $95 \%$ et p-values issus de chaque modèle logistique multivarié.

d Ind : individuels.

vement satisfaisant au vu de la durée écoulée entre les deux enquêtes. Enfin, le pourcentage de lombalgies prévalentes lors de la première enquête ne différait pas entre répondants et non-répondants à la deuxième enquête $(27,8 \%$ versus $27,1 \%$, différence non significative).
Les études longitudinales avec recueil de données continu dans le temps sont difficiles à mener sur des cohortes de travailleurs. Ainsi la majorité des études prospectives dans ce contexte adopte, comme ici, un design d'études transversales répétées sur cohorte fermée $[16,19,20]$. 
Comme la plupart des études menées en milieu de travail, nos résultats peuvent également être sujets à un biais de sélection lié à l'effet travailleur sain, ayant pu protéger les sujets les plus symptomatiques des postes à risque dès la première enquête (biais à l'inclusion), ou conduire les salariés symptomatiques à modifier leurs expositions voire à quitter leurs postes plus précocement que les autres avant la deuxième enquête (biais lors du suivi). Ce biais a néanmoins été limité par les efforts déployés pour réévaluer l'état de santé de tous les sujets initialement inclus.

Nous avons modélisé le risque de lombalgie prévalente lors de la deuxième enquête (si l'on admet une prévalence de période d'une semaine). Il a été montré que la fiabilité de la déclaration de tels symptômes diminuait avec la durée de rappel concernée, d'où notre préférence pour une période de rappel courte [21].

L'évaluation des expositions professionnelles reposait sur un auto-questionnaire. Une observation externe directe ou des mesures réalisées sur le poste de travail sont le plus souvent considérées comme les méthodes de référence. Mais si la validité des auto-questionnaires est variable selon les facteurs étudiés, celle des méthodes dites de référence est insuffisamment établie, et critiquable, du fait d'une grande variabilité intersalariée et intrasalariée au cours du temps, pour un même poste de travail, souvent non prise en compte [22]. En outre, la majorité des mesures validées d'exposition aux facteurs psychosociaux repose sur des auto-questionnaires [11].

Certains facteurs de risque de lombalgie évoqués dans la littérature n'étaient pas recueillis dans cette étude et n'ont donc pas pu être intégrés dans nos modèles de risque (tabagisme, activité physique extra-professionnelle, facteurs psychologiques individuels). Toutefois l'effet du tabagisme semble modeste, et celui des deux autres facteurs de risque cités ne sont pas consensuels $[23,24]$.

Les analyses ont été stratifiées sur le sexe. En effet, il existe des différences majeures de répartition des facteurs de risque entre les hommes et les femmes. Cette stratégie de stratification sur le sexe est privilégiée dans la littérature [25].

Afin d'évaluer l'influence des antécédents de lombalgie sur les modèles obtenus, les analyses ont été réalisées d'une part sur la totalité de la cohorte, d'autre part après exclusion des sujets présentant des lombalgies prévalentes lors de la première enquête, afin d'écarter les lombalgies chroniques ou récurrentes depuis la première enquête. Cette stratégie est d'usage courant dans les études sur ces pathologies fréquentes et récurrentes $[19,26]$.
La déclaration de douleurs du cou et/ou des MS était le facteur de risque principal de lombalgie ultérieure dans nos modèles. La valeur prédictive d'un antécédent de TMS, quel qu'il soit, sur le risque de développer un autre TMS a déjà été mise en évidence dans des travaux similaires [27]. Néanmoins, un rôle intermédiaire des douleurs du cou, entre certains facteurs de risque étudiés et la survenue de lombalgies, ne peut pas être totalement exclu, et l'ajustement sur ce facteur pourrait alors conduire à un surajustement. C'est pourquoi nos modèles ont été réalisés avec, puis sans ce facteur.

Un deuxième facteur de risque majeur était les mouvements du rachis, résultat concordant avec la littérature $[9,10]$ et les hypothèses physiopathologiques sousjacentes [28]. Se pencher en avant et sur le côté était associé à un risque de lombalgie ultérieure supérieur à celui de se pencher en avant seulement.

La conduite d'engins de chantier accroissait modérément le risque de lombalgie. L'exposition aux vibrations dites corps-entier est un facteur classique de lombalgie [9, 10,29], Ce facteur n'était toutefois significatif que dans les modèles relatifs à la cohorte entière. La réduction de l'effectif aux salariés indemnes de lombalgie initialement a pu favoriser, via une perte de puissance, la perte de significativité statistique.

'Travailler plus que prévu' semblait être un facteur de risque de lombalgie ultérieure et pourrait rendre compte de situations où le travailleur ne dispose pas des ressources suffisantes pour mener à bien sa tâche dans les temps impartis. L'enquête ESTEV 1990 avait ainsi constaté une prévalence accrue des lombalgies parmi les salariés déclarant 'ne pas avoir les moyens en temps, en matériel et en information pour faire un travail de bonne qualité' [30].

Un faible soutien de la hiérarchie apparaissait comme facteur de risque significatif de lombalgie, après retrait du facteur 'douleurs du cou et/ou des MS'. Différents mécanismes pourraient expliquer l'impact de cette variable psychosociale : modifications biomécaniques, variation dans le seuil de perception des douleurs ou encore modulation de leur déclaration [11]. Lorsque l'on s'intéressait exclusivement aux patients indemnes de lombalgie initialement, un faible soutien de la hiérarchie n'était plus associé au risque de lombalgie (y compris après retrait du facteur 'douleurs du cou et/ou des MS', données non présentées), ce qui pourrait suggérer un impact plus fort de cette variable sur le risque de récurrence des lombalgies que sur celui d'incidence, ce qui a déjà été décrit pour d'autres TMS [31].

À l'inverse, chez les salariés indemnes de lombalgie initialement, les risques liés à 'travailler plus que prévu' et 'se pencher plus de deux heures par jour' étaient 
supérieurs à ceux estimés sur la cohorte entière, ce qui pouvait suggérer un impact supérieur de ces facteurs sur l'incidence des lombalgies que sur leur récurrence.

Dans notre étude, le port de charges n'était pas associé à la déclaration ultérieure de lombalgies. Ce facteur a pourtant été largement incriminé dans la littérature [9], même si des études récentes suggèrent que l'effet pourrait être modulé par l'âge [32] ou qu'il pourrait favoriser préférentiellement les lombalgies accompagnées de douleur irradiant sous le genou [33]. La difficulté de l'autoévaluation de cette exposition (estimation simultanée de la fréquence quotidienne et du poids des charges) a probablement favorisé dans notre étude la forte proportion de données manquantes. L'absence de réponse a été ici considérée comme une modalité à part entière, en faisant l'hypothèse qu'elle correspondait à des sujets soumis à des expositions intermédiaires (irrégulières ou difficilement quantifiables selon le questionnaire). Néanmoins, un biais de classement n'est pas exclu, et il est difficile de conclure sur le rôle sur le port de charges à partir de nos données.

En conclusion, il s'agit d'une étude originale, basée sur une vaste cohorte de salariés, globalement représentative de la population salariée des Pays de la Loire, ayant mesuré de nombreux facteurs de risque afin de modéliser prospectivement le risque de lombalgie. Malgré certaines limites méthodologiques évoquées ci-dessus, nos modèles de risque se sont avérés robustes, avec une bonne constance dans la sélection de variables. Les analyses de sensibilité ont permis de formuler des hypothèses concernant les mécanismes d'action et l'interprétation de certains facteurs de risque repérés par l'analyse principale.

Les mouvements du rachis et la conduite d'engins étaient les principaux facteurs de risque biomécaniques mis en évidence. Une variable organisationnelle, 'travailler plus que prévu', probable marqueur d'intensification du travail, était également significativement liée à la présence ultérieure de lombalgie. La déclaration initiale de douleurs du cou et/ou du MS, facteur le plus fortement associé aux lombalgies, était d'interprétation délicate. En l'absence de ce dernier facteur, une variable psychosociale, le faible soutien de la hiérarchie, était liée à la prévalence ultérieure des lombalgies, et semblait avoir un impact plutôt sur le risque de récurrence, alors que les variables biomécaniques et organisationnelles citées ci-dessus paraissaient avoir un effet orienté préférentiellement sur le risque d'incidence.

Le recueil complémentaire de certains facteurs individuels et la mise en évidence de facteurs de risque plus spécifiques de certains types de lombalgie pourraient compléter et enrichir les conclusions de cette étude, qui constitue néanmoins un apport original et utile pour des actions de prévention en milieu de travail.

\section{Remerciements}

Merci aux 83 médecins du travail impliqués dans le réseau de surveillance épidémiologique des Pays de la Loire: Docteurs Abonnat, Banon, Bardet, Becquemie, Bertin, Bertrand, Bidron, Biton, Bizouarne, Boisse, Bonamy, Bonneau, Bouguer, Bourrut-Lacouture, Breton, Caillon, Cesbron, Chisacof, Chotard, Compain, Coquin-Georgeac, Cordes, Couet, Coutand, Danielou, Darcy, Davenas, De Lansalut, De Lescure, Diquelou, Dopsent, Dufrenne-Benetti, Dupas, Evano, Fache, Fontaine, Frampas Chotard, Guillier, Guillimin, Harinte, Harrigan, Hervio, Hirigoyen, Jahan, Jube, Kalfon, Labraga, Laine, Colin, Laventure, Le Clerc, Le Dizet, Lechevalier, Ledenvic, Leroux, Leroy-Maguer, Levrard, Levy, Logeay, Lucas, Mallet, Martin-Laurent, Mazoyer, Meritet, Michel, Migne, Cousseau, Moisan, Page, Patillot, Pinaud, Pineau, Pizzalla, Plessis, Plouhinec, Raffray, Robin Riom, Roussel, Russu, Saboureault, Schlindwein, Soulard, Thomson, Treillard, et Tripodi.

\section{Financement}

Merci à la Caisse nationale de l'assurance maladie des travailleurs salariés pour son soutien financier à la réalisation de cette étude.

\section{RÉFÉRENCES}

1. Henschke N, Maher CG, Refshauge KM, Herbert RD, Cumming RG, Bleasel J, et al. Prevalence of and screening for serious spinal pathology in patients presenting to primary care settings with acute low back pain. Arthritis Rheum. 2009;60(10):3072-80.

2. Gourmelen J, Chastang JF, Ozguler A, Lanoe JL, Ravaud $J F$, Leclerc A. Frequency of low back pain among men and women aged 30 to 64 years in France. Results of two national surveys. Ann Readapt Med Phys. 2007;50(8):640-4, 33-9.

3. Hart LG, Deyo RA, Cherkin DC. Physician office visits for low back pain. Frequency, clinical evaluation, and treatment patterns from a U.S. national survey. Spine. 1995;20(1):11-9.

4. Ekman M, Jonhagen S, Hunsche E, Jonsson L. Burden of illness of chronic low back pain in Sweden: a cross-sectional, retrospective study in primary care setting. Spine. 2005;30(15):1777-85.

5. Walker BF, Muller R, Grant WD. Low back pain in Australian adults: the economic burden. Asia Pac J Public Health. 2003;15(2): 79-87.

6. Ozguler A, Leclerc A, Landre MF, Pietri-Taleb F, Niedhammer I. Individual and occupational determinants of low back pain according to various definitions of low back pain. J Epidemiol Community Health. 2000;54(3):215-20.

7. Dionne CE, Dunn KM, Croft PR, Nachemson AL, Buchbinder $R$, Walker $B F$, et al. A consensus approach toward the standardization of back pain definitions for use in prevalence studies. Spine. 2008;33(1):95-103. 
8. Eisen EA. Methodology for analyzing episodic events. Scand J Work Environ Health. 1999;25 Suppl 4:36-42.

9. Burdorf A, Sorock G. Positive and negative evidence of risk factors for back disorders. Scand J Work Environ Health. 1997;23(4):243-56.

10. Lotters F, Burdorf A, Kuiper J, Miedema H. Model for the work-relatedness of low-back pain. Scand J Work Environ Health. 2003;29(6):431-40.

11. Davis $K G$, Heaney $C A$. The relationship between psychosocial work characteristics and low back pain: underlying methodological issues. Clin Biomech. 2000;15(6):389-406.

12. Ha C. Roquelaure Y. Réseau expérimental de surveillance épidémiologique des troubles musculo-squelettiques dans les Pays de la Loire - Protocole de la surveillance dans les entreprises (2002-2004). Saint-Maurice: Institut de veille sanitaire ; $2007.84 p$

13. Kuorinka I, Jonsson B, Kilbom A, Vinterberg H, BieringSorensen F, Andersson G, et al. Standardised Nordic questionnaires for the analysis of musculoskeletal symptoms. Appl Ergon. 1987;18(3):233-7.

14. Niedhammer I. Psychometric properties of the French version of the Karasek Job Content Questionnaire: a study of the scales of decision latitude, psychological demands, social support, and physical demands in the GAZEL cohort. Int Arch Occup Environ Health. 2002;75(3):129-44.

15. Larocque B, Brisson C, Blanchette C. Cohérence interne, validité factorielle et validité discriminante de la traduction française des échelles de demande psychologique et de latitude décisionnelle du "Job Content Questionnaire » de Karasek. Rev Épidemiol Santé Publique. 1998;46(5):371-81.

16. Plouvier S, Leclerc A, Chastang JF, Bonenfant S, Goldberg M. Socioeconomic position and low-back pain - the role of biomechanical strains and psychosocial work factors in the GAZEL cohort. Scand J Work Environ Health. 2009;35(6):42936.

17. Hosmer DW, Lemeshow S. Applied Logistic Regression. In: Interscience W, editor. Second ed 2000. p. 92-104.

18. Goldberg M, Luce D. Les effets de sélection dans les cohortes épidémiologiques Nature, causes et conséquences. Rev Epidemiol Sante Publique. 2001;49(5):477-92.

19. Andersen JH, Haahr JP, Frost P. Risk factors for more severe regional musculoskeletal symptoms: a two-year prospective study of a general working population. Arthritis Rheum. 2007;56(4):1355-64.

20. Leclerc A, Landre MF, Chastang JF, Niedhammer I, Roquelaure Y. Upper-limb disorders in repetitive work. Scand $J$ Work Environ Health. 2001;27(4):268-78.
21. Miranda H, Gold JE, Gore R, Punnett L. Recall of prior musculoskeletal pain. Scand J Work Environ Health. 2006;32(4):294-9.

22. Stock SR, Fernandes R, Delisle A, Vezina N. Reproducibility and validity of workers' self-reports of physical work demands. Scand J Work Environ Health. 2005;31(6):409-37.

23. Shiri R, Karppinen J, Leino-Arjas P, Solovieva S, ViikariJuntura E. The association between smoking and low back pain: a meta-analysis. Am J Med. 2010;123(1):87 e7-35.

24. Goldberg MS, Scott SC, Mayo NE. A review of the association between cigarette smoking and the development of nonspecific back pain and related outcomes. Spine. 2000;25(8):995-1014.

25. Messing K, Stock SR, Tissot F. Should studies of risk factors for musculoskeletal disorders be stratified by gender? Lessons from the 1998 Quebec Health and Social Survey. Scand J Work Environ Health. 2009;35(2):96-112.

26. Hoogendoorn WE, Bongers PM, de Vet HC, Houtman IL, Ariens GA, van Mechelen W, et al. Psychosocial work characteristics and psychological strain in relation to low-back pain. Scand J Work Environ Health. 2001;27(4):258-67.

27. Cassou B, Derriennic F, Monfort C, Norton J, Touranchet $A$. Chronic neck and shoulder pain, age, and working conditions: longitudinal results from a large random sample in France. Occup Environ Med. 2002;59(8):537-44.

28. Kumar, ed. Biomechanics in ergonomics. London: Taylor \& Francis; 2008.

29. Tiemessen IJ, Hulshof CT, Frings-Dresen MH. Low back pain in drivers exposed to whole body vibration: analysis of a dose-response pattern. Occup Environ Med. 2008;65(10):66775 .

30. Derriennic F. TA, Volkoff S. Âge, travail, santé - étude sur les salariés âgés de 37 à 52 ans - Enquête ESTEV 1990. Paris : Les éditions INSERM; 1996. p. 159-79.

31. Bongers PM, Ijmker $S$, van den Heuvel $S$, Blatter BM. Epidemiology of work related neck and upper limb problems: psychosocial and personal risk factors (part I) and effective interventions from a bio behavioural perspective (part II). J Occup Rehabil. 2006;16(3):279-302.

32. Miranda H, Viikari-Juntura E, Punnett L, Riihimaki $H$. Occupational loading, health behavior and sleep disturbance as predictors of low-back pain. Scand J Work Environ Health. 2008;34(6):411-9.

33. Plouvier S, Renahy E, Chastang JF, Bonenfant S, Leclerc A. Biomechanical strains and low back disorders: quantifying the effects of the number of years of exposure on various types of pain. Occup Environ Med. 2008;65(4):268-74. 